\title{
Evidence for the Double Excimer State of conjugated polymer in a liquid solution
}

\section{K. H. Ibnaouf}

\section{S. Prasad}

V. Masilamani masila123@gmail.com

\section{S. AlSalhi}

\section{A. S. Alaamer}

\begin{abstract}
Al-Imam Mohammed bin Saud Islamic University, Physics Department, College of Science P.O. Box 90950, Riyadh 11623, Saudi Arabia

KSU Research Chair on Laser Diagnosis of Cancers, King Saud University, Physics Department and Astronomy, College of Science, Riyadh 11623, Saudi Arabia

KSU Research Chair on Laser Diagnosis of Cancers, King Saud University, Physics Department and Astronomy, College of Science, Riyadh 11623, Saudi Arabia

KSU Research Chair on Laser Diagnosis of Cancers, King Saud University, Physics Department and Astronomy, College of Science, Riyadh 11623, Saudi Arabia

Al-Imam University, Physics Department, College of Science P.O. Box 90950, Riyadh 11623, Saudi Arabia
\end{abstract}

In this paper, the spectral properties of a conjugated polymer poly [2-methoxy-5-(2-ethylhexyloxy)-1, 4-phenylenevinylene] (MEH-PPV) in benzene have been studied. The results showed that the fluorescence spectra of MEH-PPV under low concentrations had two peaks; the dominant one due to monomer was around $560 \mathrm{~nm}$, and the shoulder one attributed to the excimer was around $600 \mathrm{~nm}$. Under higher concentrations, it was found that there was only one band around $600 \mathrm{~nm}$ due to the excimeric state. By increasing the concentrations of MEH-PPV, it could be seen that there was a new band around $640 \mathrm{~nm}$. This band is being attributed to the double excimer. Under high power pulsed laser excitation, we observed amplified spontaneous emission (ASE) at $570 \mathrm{~nm}, 605 \mathrm{~nm}$ and $650 \mathrm{~nm}$. These ASE peaks could arise from the monomer, excimer and double excimer states of the macromolecule respectively. To the best of our knowledge this is perhaps the first report on ASE from double excimer of the conjugated polymer, MEH-PPV in liquid solution.

[DOI: http://dx.doi.org/10.2971/jeos.2013.13001]

Keywords: Conjugated polymer MEH-PPV, fluorescence, double excimer, ASE from double excime

\section{INTRODUCTION}

Many conjugated polymers are capable of light emission [1]. This can be generated either optically, by shining light onto a sample to give fluorescence, or electrically, by applying a voltage to a polymer light-emitting diode [2].

The excited states that exist in conjugated polymers by charge injection or photo excitation have photo physical characteristics similar to those of small organic chromophores such as laser dyes [3]. There are several reasons why semiconducting polymers could be attractive laser materials [4]-[6]: The first is that there exists a range of polymers that can emit light across the visible spectrum. Secondly, these polymers have broad spectra suitable for tunable lasers. They have very strong absorption and emission cross section, which implies that there is the potential for the strong amplification of light. Thirdly, the absorption and fluorescence spectra are well separated, so that re -absorption of emitted light is weak. Fourthly, in many fluorescent organic molecules (including laser dyes), light emission is severely quenched at high concentrations such as that typically found in solid films. In contrast, conjugated polymers can emit light as neat solid films and, in addition, are capable of charge transport, thereby providing the potential to make electrically pumped lasers in the not too distant future.
The laser action of a semiconducting polymer MEH-PPV in the liquid state, operating in the yellow/ red wavelength region, was achieved for the first time in 1992. The reported lasing performance of this polymer was comparable to that of the most efficient laser dye, Rhodamine 6G [7]. The lasing performance and wavelength tunability of a novel high efficiency copolymer TOP-PPV was achieved for the first time in 1995. The reported lasing of this polymer was comparable to those of Coumarin 120 and Coumarin 47 [8].

The spectral properties of MEH-PPV in different solvents and different concentrations had been described in a preceding paper by us. The results showed that the conjugated conducting polymer MEH-PPV could exist in the excimer state in a few solutions [9]. The amplified spontaneous emission characteristics (ASE) of MEH-PPV in different solvents and at different concentrations were studied under pulsed-laser excitation at $355 \mathrm{~nm}$ and $532 \mathrm{~nm}$; the conversion efficiency of MEH-PPV is comparable to other conventional laser dyes Rhodamine $\mathrm{B}$ and Rhodamine 6G [10].

In this paper, the spectral properties of a conjugated polymer, MEH-PPV in benzene with different concentrations and temperatures are described. The results showed that under suf- 


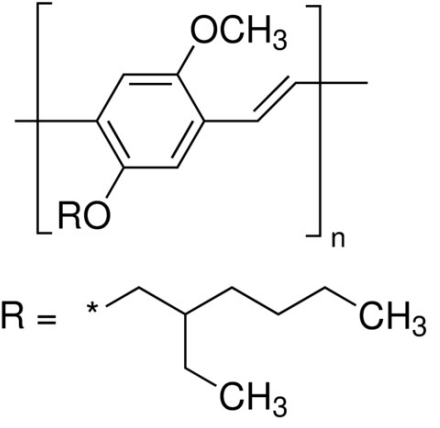

FIG. 1 Molecular Structures of polymer of MEH-PPV.

ficient concentration, this conjugated polymer could exhibit double excimer formation with a fluorescence peak at $640 \mathrm{~nm}$. At this concentration, with sufficient pump power, the ASE with a peak at $650 \mathrm{~nm}$ with FWHM of $8 \mathrm{~nm}$ also could be obtained.

\section{EXPERIMENTAL}

MEH-PPV was purchased from Sigma-Aldrich. The molecular structure is given in Figure 1. This is a macromolecule with a molecular weight of 70000-100000. The absorption and fluorescence spectra of MEH-PPV in benzene were studied under wide range of concentrations. The spectra for the solutions were measured in a quartz cuvette with the dimensions $1 \times 1 \times 4 \mathrm{~cm}$ with an optical path length of $1 \mathrm{~cm}$.

UV-VIS absorption spectra were taken using a Perkin Elmer spectrophotometer and the fluorescence was measured on a Perkin Elmer LS45 spectrofluorometer.

The third harmonic of an Nd: YAG laser (355 nm) Quantel Inc., with a pulse width of $6 \mathrm{~ns}$, was used as the excitation source. The UV laser was focused by a quartz cylindrical lens with a focal length of $5 \mathrm{~cm}$. This was used to do transverse excitation of the MEH-PPV solution taken in a four-side polished quartz cell, which was kept canted to avoid feedback. See reference [10] for more details. At optimum values of the pump power and concentrations of MEH-PPV, we observed an ASE beam coming out as a cone of light. This was collected by a $1 \mathrm{~mm}$ entrance slit of an ICCD camera, which displayed the spectra of the ASE.

\section{RESULTS AND DISCUSSION}

\subsection{Excimer State of MEH-PPV}

\subsubsection{Spectral Properties:}

The absorption spectra of MEH-PPV in benzene were recorded for a wide range of concentrations from 1.0 to $8.0 \mu \mathrm{M}$. It was found that there were two peaks: one around $333 \mathrm{~nm}$ and the other around $500 \mathrm{~nm}$; the shape of the absorption spectra remained the same irrespective of the concentration, although the optical density increased with an increasing concentration of MEH-PPV as shown in Figure 2.

The fluorescence spectra of MEH-PPV in benzene at low con-

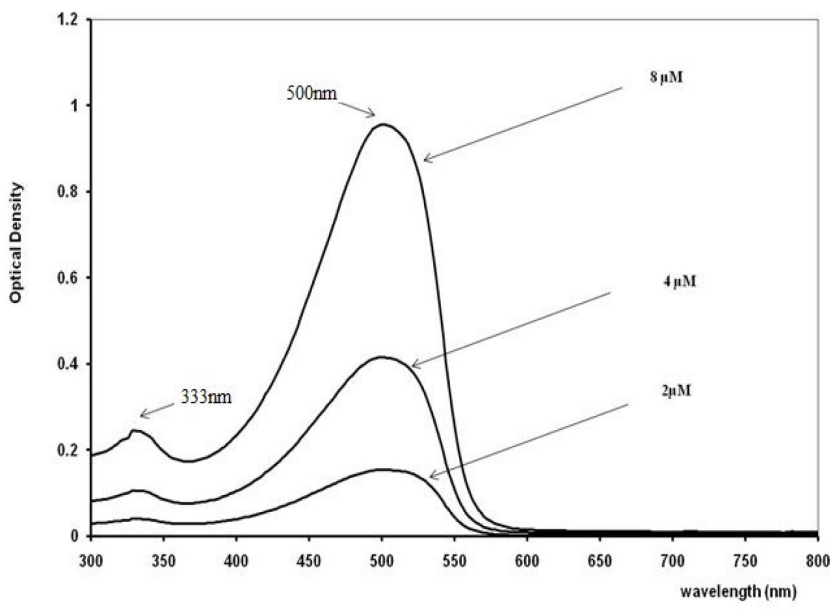

FIG. 2 Absorption spectra of MEH-PPV in benzene at different concentrations.

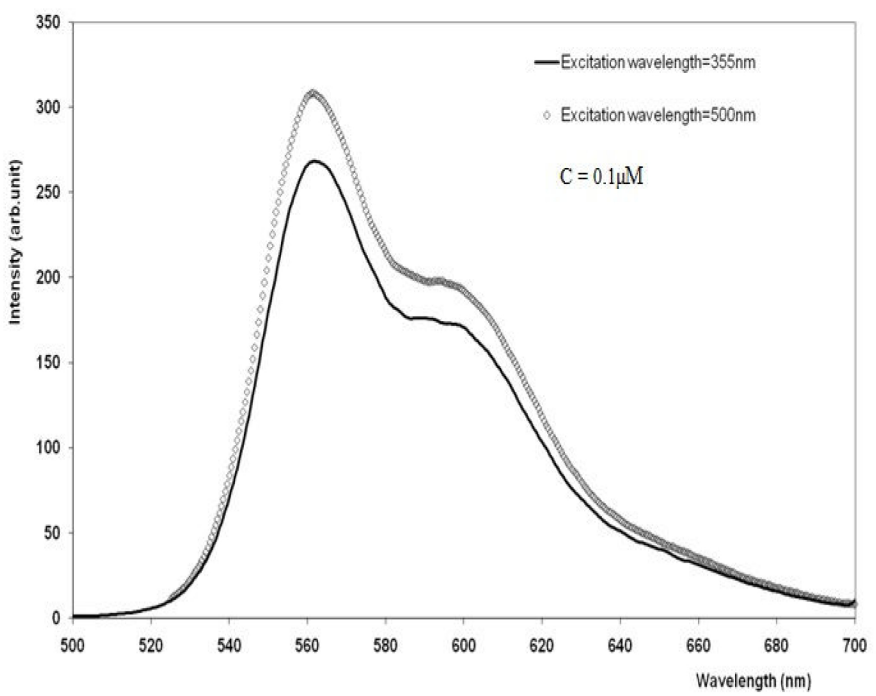

FIG. 3 Fluorescence spectra of MEH-PPV in benzene at two different wavelengths of excitations ( 355 \& $500 \mathrm{~nm}$ ).

centration $(1 \mu \mathrm{M})$ were recorded. The result showed that there were two peaks of the fluorescence: one around $560 \mathrm{~nm}$, and other at $600 \mathrm{~nm}$; the shape of the fluorescence spectra did not change for two different wavelengths of excitation $(355 \mathrm{~nm}$ and $500 \mathrm{~nm}$ ), as shown in Figure 3, indicating that there was only one emitting species for MEH-PPV in benzene at low concentrations.

For high concentrations from 0.1 to $10.0 \mu \mathrm{M}$ of MEH-PPV in benzene, the fluorescence spectra were dramatically different. It can be seen that when the concentration was increased up $1.0 \mu \mathrm{M}$, the result showed that the band around $600 \mathrm{~nm}$, which was weaker at $0.1 \mu \mathrm{M}$, became comparable to that at $560 \mathrm{~nm}$. At concentrations greater than $1.0 \mu \mathrm{M}$, the band around 600 $\mathrm{nm}$ became dominating, and the band around $560 \mathrm{~nm}$ was almost absent as shown in Figure 4. It means that MEH-PPV in benzene exists in the excimeric state [9].

Figure 5 shows the variation in the fluorescence intensities for $\mathrm{MEH}-\mathrm{PPV}$ in benzene at $0.4 \mu \mathrm{M}$ for different temperatures. The results show that the fluorescence due to the excimer is $75 \%$ of the fluorescence due to the monomer at room temper- 


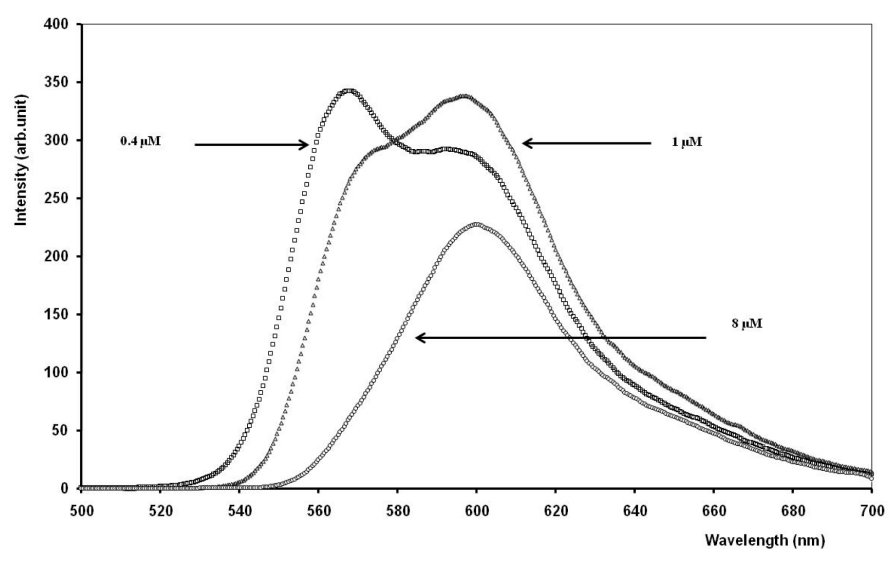

FIG. 4 Fluorescence spectra of MEH-PPV in benzene at different concentrations.

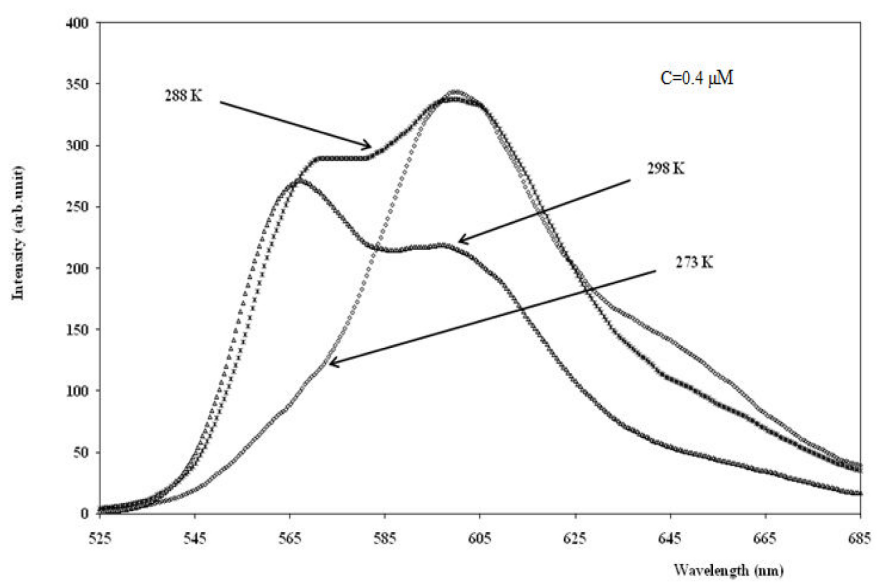

FIG. 5 Fluorescence of MEH-PPV in benzene at $0.4 \mu \mathrm{M}$ for different temperatures.

ature $\left(25^{\circ} \mathrm{C}\right)$; but, as the temperature was increased, the intensities of both bands decreased, although the band around $600 \mathrm{~nm}$ fell faster. On the other hand, when the temperature was decreased, the intensities of both bands increased, although the band around $600 \mathrm{~nm}$ grew much faster, and at $0^{\circ} \mathrm{C}$, there was just only one band, which was around $600 \mathrm{~nm}$; the band around $560 \mathrm{~nm}$ was almost absent. This is the general trend for any excimer [9].

\subsubsection{ASE Spectrum:}

The ASE spectrum of MEH-PPV in benzene at a concentration of $10 \mu \mathrm{M}$ was studied. This solution was excited using the third harmonic of Nd: YAG laser $(\lambda=355 \mathrm{~nm})$. The results showed that the ASE peak was found around $600 \mathrm{~nm}$ with a narrow spectral bandwidth of $3 \mathrm{~nm}$ (FWHM). This peak coincided with the maximum of the fluorescence emission spectrum at this concentration, as shown in Figure 6. One can immediately see that the ASE with high degree of optical gain and hence the laser action had been produced from the excimeric state [10].

\subsection{Double Excimer State (MEH-PPV)}

\subsubsection{Spectral Properties:}

To study the spectral properties of MEH-PPV at still higher concentrations, MEH-PPV was dissolved for different con-

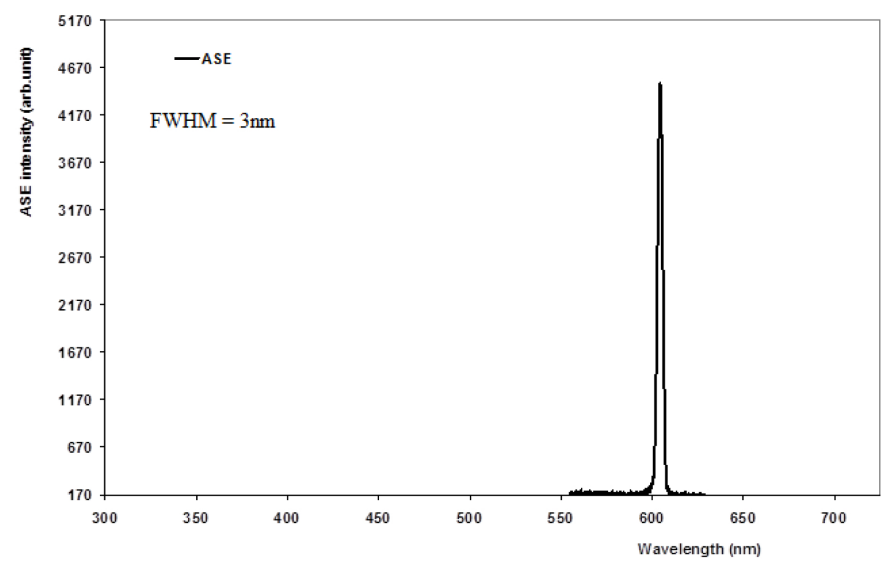

FIG. 6 ASE spectra of MEH-PPV in benzene at $10 \mu \mathrm{M}$.

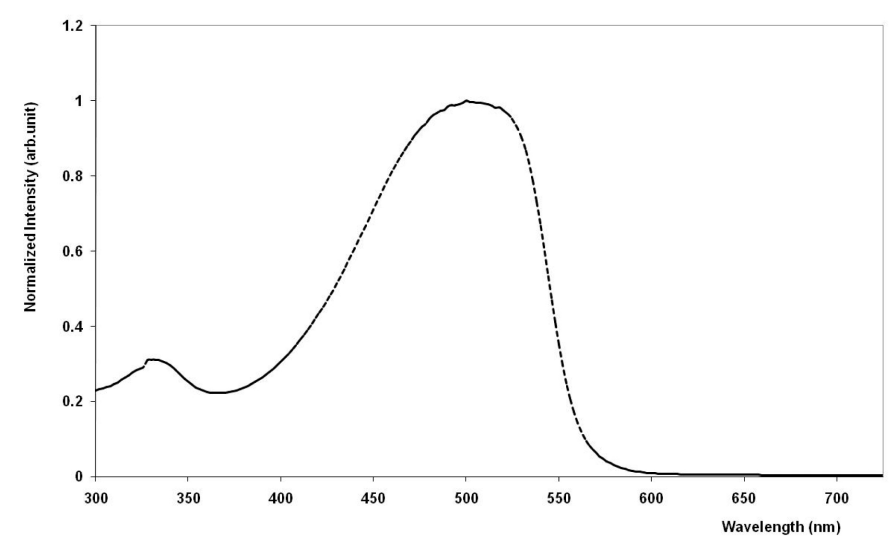

FIG. 7 Absorption spectra of MEH-PPV in benzene at $300 \mu \mathrm{M}$.

centrations from 10 to $300 \mu \mathrm{M}$. The absorption spectra of MEH-PPV in benzene under all of these concentrations were recorded using a thin film of solution in the cuvette. The experimental results showed the presence of two peaks of absorption spectra as reported earlier [9] and one around $333 \mathrm{~nm}$ and the other around $500 \mathrm{~nm}$. The shape of the absorption spectra did not change for all these concentrations indicating the absence of ground state aggregation. It was observed that the optical density increased with an increasing concentration of MEH-PPV as shown in Figure 7. Note the ordinates are displayed only as normalized intensity in arbitrary units.

The fluorescence spectra also were recorded for the above set of solutions. At concentration $10 \mu \mathrm{M}$; there was only one band around $600 \mathrm{~nm}$ which was due to the excimer and the peak around $560 \mathrm{~nm}$ almost disappeared, as mentioned earlier, (see Figure 4). When the concentration increased up to $100 \mu \mathrm{M}$, it was found that the peak due to the excimer was red shifted to $610 \mathrm{~nm}$; this red shift may be due to the re-absorption process, and at the long wavelength end of the spectrum there was a small hump around $640 \mathrm{~nm}$ (as shown in Figure 8). This peak around $640 \mathrm{~nm}$ is a new peak and did not exist for low concentrations. This is attributed the double excimeric state of MEH-PPV.

By increasing the concentration to $200 \mu \mathrm{M}$, the peak around $610 \mathrm{~nm}$ (which due to the excimer) was shifted to be around $615 \mathrm{~nm}$ and the intensity decreased, while the intensity of the peak at $640 \mathrm{~nm}$, attributed to the double excimer, increased 


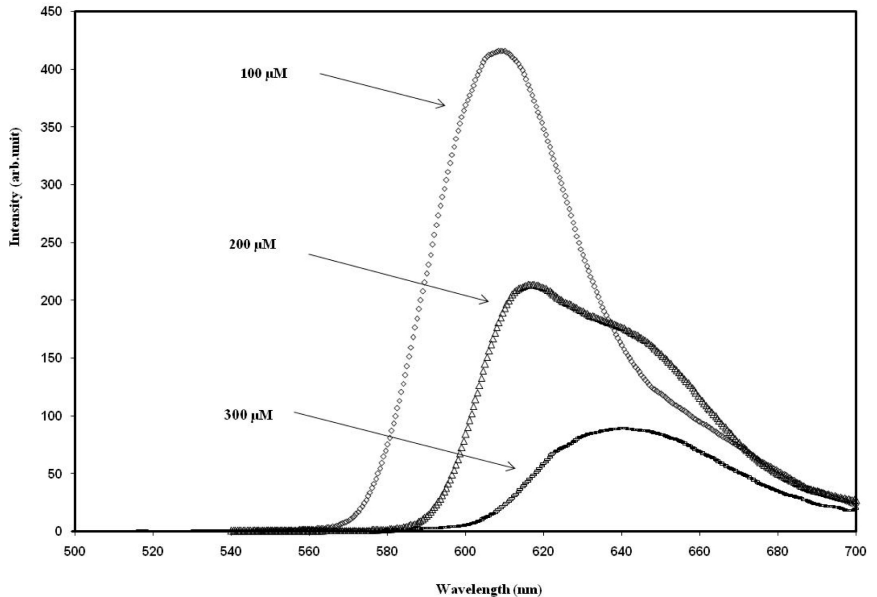

FIG. 8 Fluorescence spectra of MEH-PPV in benzene at different higher concentrations.

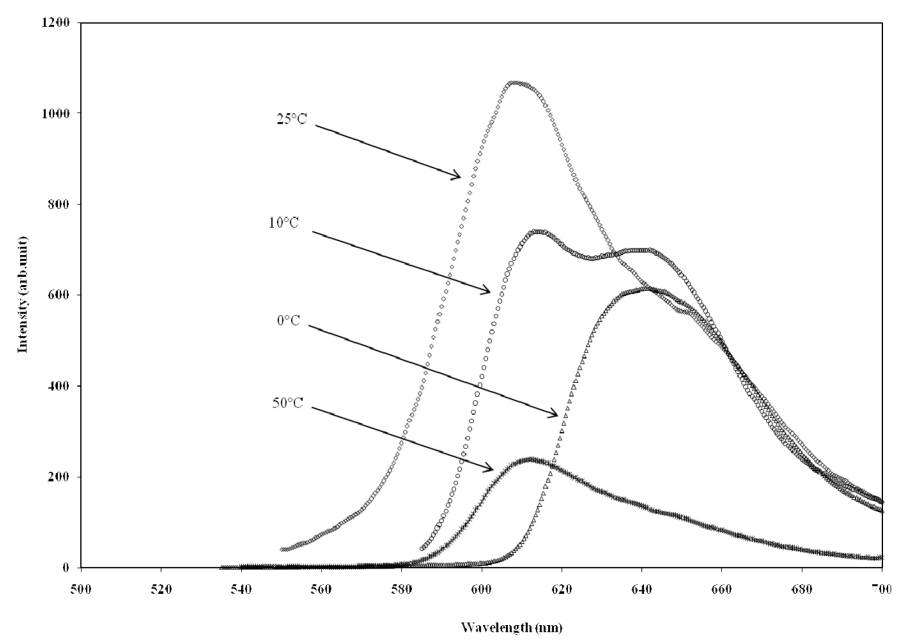

FIC. 9 Fluorescence of MEH-PPV in benzene at $100 \mu \mathrm{M}$ for different temperatures.

a little bit. The fluorescence due to the double excimer was $75 \%$ of the fluorescence due to the excimer at room temperature $\left(25^{\circ} \mathrm{C}\right)$, see Figure 8 . At concentration $300 \mu \mathrm{M}$, the peak around $615 \mathrm{~nm}$ disappeared and there was only one dominant peak around $640 \mathrm{~nm}$ as shown in Figure 8 indicating the double excimeric state of MEH-PPV.

The effect of temperature on the fluorescence intensities of MEH-PPV in benzene at $100 \mu \mathrm{M}$ was also studied. It could be seen that the intensity at $640 \mathrm{~nm}$ (due to double excimer state) was only $50 \%$ of the intensity at $615 \mathrm{~nm}$ (due to single excimer) at the room temperature $\left(25^{\circ} \mathrm{C}\right)$; but, when the temperature was increased, the intensities of both bands decreased, although the band around $640 \mathrm{~nm}$ was about to vanish. On the other hand, when the temperature was decreased, the intensities of both bands increased, although the band around $640 \mathrm{~nm}$ grew much faster, and at $10^{\circ} \mathrm{C}$, the peak at $640 \mathrm{~nm}$ became comparable to that at $615 \mathrm{~nm}$; at $0^{\circ} \mathrm{C}$, there was only one band around $640 \mathrm{~nm}$ and the band around $615 \mathrm{~nm}$ almost disappeared as shown in Figure 9. This is the confirmation that MEH-PPV could exist in double excimeric state. There is a one-to-one correspondence between the dynamics of monomer excimer (as in Figure 4 and 5) and double excimer

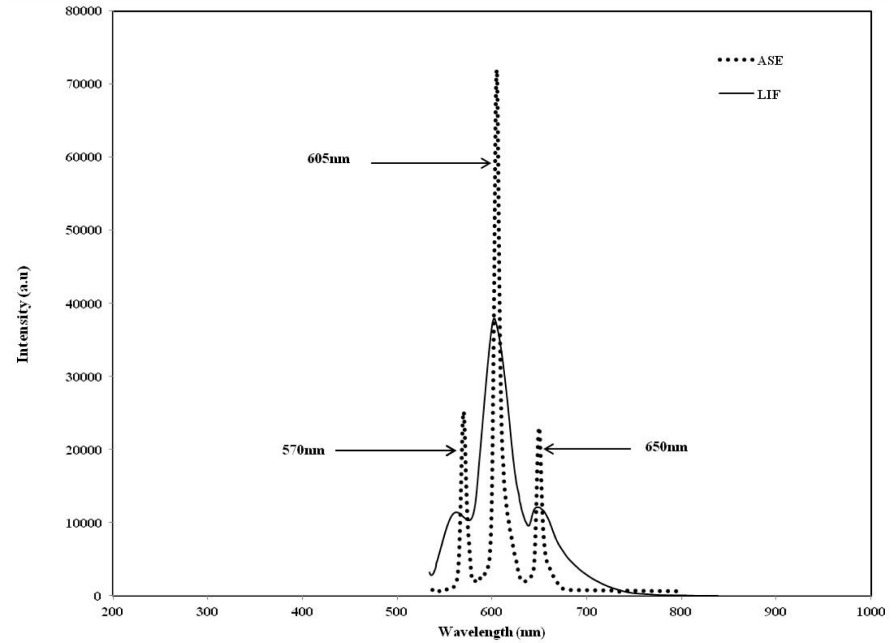

FIG. 10 ASE spectra of MEH-PPV in benzene at $300 \mu \mathrm{M}$.

(as in Figure 8 and 9). These trends give strong evidence to our hypothesis of double excimer of MEH-PPV.

\subsubsection{ASES Spectra:}

The MEH-PPV solution $(300 \mu \mathrm{M})$ in benzene was transversely excited with a UV laser at $355 \mathrm{~nm}(1 \mathrm{~mJ})$ and the laser induced fluorescence (LIF) was recorded. It could be seen that there were three bands at $570 \mathrm{~nm}, 605 \mathrm{~nm}$ and $650 \mathrm{~nm}$ with FWHM of $30 \mathrm{~nm}$ (see Figure 10).

As shown in Figure 10, the ASE occurred primarily at $600 \mathrm{~nm}$ due to the excimeric state; but there were two peaks one at $570 \mathrm{~nm}$ and other at $650 \mathrm{~nm}$ with FWHM of each $8 \mathrm{~nm}$ which were due to the monomer and the double excimer respectively at a pump power of $50 \mathrm{~mJ}$. Note the one-to-one correspondence between the steady state fluorescence shown in Figure 8, the LIF and the ASE shown in Figure 10.

It is important to mention that any molecule, under higher concentration undergoes a high level of quenching and fluorescence from excimer is rather weak compared to the monomer. The situation gets even worse for the double excimer as shown in Figure 8 and 9. In the concentration range of $100 \mu \mathrm{M}-300 \mu \mathrm{M}$, the excimer and double excimer are in competition as much as the monomer and the excimer in the range $0.5-5.0 \mu \mathrm{M}$. However, this particular molecule exhibits easier tendency to form excimer [10] but not double excimer. Because of these two reasons, the number of molecule existing in the double excimer state and stimulated emission cross section of both could be far smaller than the (single) excimeric state. This could be the reason why we got ASE at $600 \mathrm{~nm}$ mostly due to the (single) excimer and only as a small peak at $650 \mathrm{~nm}$ due to double excimer. Also note another shoulder at $570 \mathrm{~nm}$ due to the monomer. We had shown earlier that though monomer emission was easier and stronger in steady state fluorescence, it was the excimer overwhelming in optical gain spectra, something parallel to laser Raman and Raman laser dynamics.

At this point we could rather draw attention to the report of Samuel et al [11] of laser action at $640 \mathrm{~nm}$ from the same ma- 
terial with a distributed feedback configuration achieved by a corrugation embedded on a thin film. This also could be due to the double excimer of MEH-PPV.

\section{CONCLUSION}

Excimer is generally rare, either in gaseous or liquid states. The laser action from such excimer is still very rare. Under these circumstances, we have been able to show evidence for the existence of double excimer from the steady state fluorescence and laser induced florescence (LIF) of this new molecular species. This paper also shows the newly hypothesized double excimer (from MEH-PPV in benzene solution).has adequate optical gain, capable of producing ASE under pulsed laser excitation.

\section{ACKN OWLEDGEMENT}

This project was supported by King Saud University, Deanship of Scientific Research, College of Science Research Center.

\section{References}

[1] N. Tessler, "Lasers Based on Semiconducting Organic Materials," Adv. Mater. 11 (5), 363-370 (1999).

[2] M. D. McGehee, and A. J. Heeger, "Semiconducting (Conjugated) Polymers as Materials for Solid-State Lasers," Adv. Mater. 12 (22), 1655-1668 (2000).
[3] C. Kallinger, M. Hilmer, A. Haugeneder, M. Perner, W. Spirkl, U. Lemmer, J. Feldmann, et al., "A Flexible Conjugated Polymer Laser," Adv. Mater. 10 (12), 920-923 (1998).

[4] G. Kranzelbinder, and G. Leising, "Organic Solid-State Lasers," Rep. Prog. Phys. 63 (5), 729-762 (2000).

[5] U. Scherf, S. Riechelb, U. Lemmerb, and R. F Mahrtc, “Conjugated polymers: lasing and stimulated emission," Curr. Opin. Solid. St. M. 5, 143-154 (2001).

[6] W. Holzer, A. Penzkofer, T. Pertsch, N. Danz, A. Bräuer, E. B. Kley, H. Tillmann, et al., "Corrugated neat thin-film conjugated polymer distributed feedback lasers," Appl. Phys. A-Mater. 74 (4-5), 333-342 (2002).

[7] D. Moses, "High quantum efficiency luminescence from a conducting polymer in solution: A novel polymer laser dye," Appl. Phys. Lett. 60 (26), 3215-3216 (1992).

[8] H. J. Brouwer, V. V. Krasnikov, T. A. Pham, R. E. Gill, and G. Hadziioannou, "Stimulated emission from vacuum-deposited thin films of a substituted oligo(p-phenylene vinylene)," Appl. Phys. Lett. 73 (6), 708-710 (1998).

[9] M. S. Alsalhi, K. H. Ibnaouf, V. Masilamani, and O. A. Yassin, "Excimer State of a Conjugate Polymer (MEH-PPV) in Liquid Solutions," Laser Phys. 17 (12), 1361-1366 (2007).

[10] V. Masilamani, K. H. Ibnaouf, M. S. Alsalhi, and 0. A.Yassin, "Laser Properties of a Conjugate Polymer (MEH-PPV) in the LiquidExcimeric State," Laser Phys. 17 (12), 1367-1373 (2007).

[11] D. W. Samuel, and G. A. Turnbull, "Polymer lasers: recent advances," Mater. Today 7, 28-35 (2004). 